\title{
Comparison of clinical characteristics between lean and obese nonalcoholic fatty liver disease in the northeast Chinese population
}

Hui Li ${ }^{1}$, Yanzhen Chen², Xing Tian³ ${ }^{3}$ Yu Hong ${ }^{2}$, Chengliang Chen², Nawaz Khukhar Sharokh², Jian Jiao $^{2}$

\begin{abstract}
${ }^{1}$ Dental Department, China-Japan Union Hospital, Jilin University, Changchun, China ${ }^{2}$ Department of Gastroenterology and Hepatology, China-Japan Union Hospital, Jilin University, Changchun, China
\end{abstract}

${ }^{3}$ Department of Infectious Disease, Public Health Clinical Medical Centre, Chengdu, China

Submitted: 26 March 2019

Accepted: 30 June 2019

Arch Med Sci Atheroscler Dis 2019; 4: e191-e195 DOI: https://doi.org/10.5114/amsad.2019.87122 Copyright (c) 2019 Termedia \& Banach

\section{Abstract}

Introduction: Nonalcoholic fatty liver disease (NAFLD) is seen commonly in patients with obesity, but there are many non-obese people who also have NAFLD, which we call lean NAFLD. The objective of this study is to compare the characters of lean and obese NAFLD individuals aimed to better understand the pathogenesis of NAFLD.

Material and methods: A total of 496 NAFLD cases (history of alcohol consumption, medication used within the last 3 months, hepatitis virus infection, autoimmune or hereditary liver disease were excluded), divided into an obese (body mass index (BMI) $\left.\geq 25 \mathrm{~kg} / \mathrm{m}^{2}\right) \operatorname{NAFLD~group~}(n=395)$ and lean $\left(\mathrm{BMI}<25 \mathrm{~kg} / \mathrm{m}^{2}\right)$ NAFLD group $(n=101)$, were investigated. Gender, age, alanine aminotransferase, triglycerides, cholesterol, and the blood glucose were compared between the two groups.

Results: A male-dominated discrepancy of gender distribution was more evident in obese NAFLD group. Elevated alanine amonotransferase was more common in the obese NAFLD group. 50-59 years was the peak age of both lean and obese NAFLD groups. Normal triglycerides (TG) patients were more common in the lean NAFLD group, while the proportion of elevated TG patients was higher in the obese NAFLD group. No statistically significant difference in the proportion of elevated cholesterol patients was found between the two groups. In the lean NAFLD group, the proportion of females was higher in the normal fasting glucose group.

Conclusions: Sexual dimorphism exists in lean NAFLD patients, but this trend was most pronounced during the age range 40-49 years and disappeared after entering the 50-59-year-old age range. In lean NAFLD patients, normal levels of TG and blood glucose were more common, and occurrence of non-alcoholic steatohepatitis was less common than among obese NAFLD.

Key words: lean, obese, nonalcoholic fatty liver disease, Chinese.

\section{Introduction}

Non-alcoholic fatty liver disease (NAFLD) is a non-infectious liver disease closely related to liver cirrhosis and cancer; it is seen commonly in patients with metabolic abnormalities associated with obesity, especially those with diabetes, dyslipidaemia, and metabolic syndrome.
Corresponding author:

Jian Jiao

Department

of Gastroenterology

and Hepatology

China-Japan

Union Hospital

Jilin University

Changchun, China

E-mail: jjian@jlu.edu.cn 
However, not all obese subjects develop NAFLD, and, more importantly, NAFLD also develops in lean individuals, which we call lean NAFLD. The risk factors of lean NAFLD remain poorly defined. Sexual dimorphism, age or postmenopausal status, serum level of lipid profile, and blood glucose were associated with the risk of NAFLD [1], Comparing the clinical features of obese NAFLD and lean NAFLD helps us better understand the pathogenesis of NAFLD.

\section{Material and methods}

\section{Patients}

One thousand six hundred and eight cases of physical examination were selected from the Physical Examination Centre of the China-Japan Union Hospital of Jilin University between 2015 and 2016. All cases accepted abdominal ultrasound and FibroScan examination. History of alcohol consumption and medication used within the preceding 3 months were investigated. Serum antibodies of hepatitis virus A, B, C, and E were tested. Autoimmune and hereditary liver disease were also excluded. Finally, 496 cases diagnosed with NAFLD were enrolled in this study. The detailed diagnostic criteria were according to the guidelines for NAFLD management formulated by the Chinese National Workshop on Fatty Liver Disease in 2010 [2]. NAFLD was diagnosed according to the following criteria: (1) alcohol consumption $<140 \mathrm{~g} /$ week for male adults and < $70 \mathrm{~g} /$ week for female adults; (2) absence of viral hepatitis (hepatitis B virus (HBV)/hepatitis C virus (HCV)), hepatolenticular degeneration, autoimmune diseases, a history of total parenteral nutrition, or intake of any hepatotoxic drugs (e.g. tamoxifen, amiodarone, sodium valproate, methotrexate, and glucocorticoid); and (3) ultrasonographic examination suggesting fatty infiltration in the liver and controlled attenuation parameter (CAP) $\geq 240$ $\mathrm{db} / \mathrm{m}$.

\section{Study designs}

The NAFLD patients were divided into two groups according to $\mathrm{BMI}$ : an obese NAFLD group $\left(\mathrm{BMI} \geq 25 \mathrm{~kg} / \mathrm{m}^{2}\right.$ ) and a lean NAFLD group (BMI $<25 \mathrm{~kg} / \mathrm{m}^{2}$ ). Gender, age, alanine aminotransferase (ALT), triglycerides (TG), cholesterol (CHOL), and the level of blood glucose were compared between two groups.

\section{Statistical analysis}

All data were expressed as mean \pm SD. $t$ tests were used for numerical variables and $\chi^{2}$ tests were used for categorical variables. $P$-values $<0.05$ were considered statistically significant.

\section{Results}

\section{Comparison of gender distribution and elevated ALT occurrence between the lean NAFLD group and the obese NAFLD group}

The proportion of males was higher than females in both the lean NAFLD (58.42\% vs. $41.58 \%)$ and obese NAFLD group (75.19\% vs. $24.81 \%$ ), but this male-dominated discrepancy of gender distribution was more evident in the obese NAFLD group, and a statistically significant difference was found if compared with the lean NAFLD group $(p=0.0008)$. Elevated ALT was more common in the obese NAFLD group (69.11\% vs. $30.89 \%)$ compared with the lean NAFLD group $(79.21 \%$ vs. 20.79\%) ( $p=0.0456)$ (Table I).

\section{Comparison of age distribution \\ and trend between the lean NAFLD group and the obese NAFLD group}

50-59 years was the peak age of both lean and obese NAFLD, and there was no significant difference in gender distribution during this period $(p>0.05)$. While statistically significant differences in gender distribution were found in the 40-49-year-old age group, the proportion of males was higher than that of females, regardless of lean NAFLD $(p=0.0784)$ or obese NAFLD $(p=0.0291)$ (Table II, Figure 1).

\section{Comparison of serum levels of TG between the lean NAFLD group and the obese NAFLD group}

To avoid the effects of $\mathrm{CHOL}$ and low-density lipoprotein cholesterol (LDL-C), 302 cases of NAFLD patients with normal CHOL and LDL-C were elected to analyse the difference of TG level between the lean NAFLD group and the obese NAFLD group (Table III).

Table I. Comparison of gender distribution and elevated ALT occurrence between the lean NAFLD group and the obese NAFLD group

\begin{tabular}{|lccc|}
\hline Parameter & Lean NAFLD $(n=101)$ & Obese NAFLD $(n=395)$ & $P$-value \\
\hline Male/female, $n(\%)$ & $59 / 42(58.42 / 41.58)$ & $297 / 98(75.19 / 24.81)$ & 0.0008 \\
\hline Normal ALT*/elevated ALT, $n(\%)$ & $80 / 21(79.21 / 20.79)$ & $273 / 122(69.11 / 30.89)$ & 0.0456 \\
\hline
\end{tabular}

${ }^{\star}$ Normal value of ALT: 0 40 IU/l. 
Table II. Comparison of age distribution between the lean NAFLD group and the obese NAFLD group

\begin{tabular}{|lcccc|}
\hline Age [year] & \multicolumn{2}{c}{ Lean NAFLD $(n=101)$} & \multicolumn{2}{c|}{ Obese NAFLD $(n=395)$} \\
\cline { 2 - 5 } & Female $(n=42)$ & Male $(n=59)$ & Female $(n=42)$ & Male $(n=59)$ \\
\hline $20-29$ & $4(9.52 \%)$ & $4(6.78 \%)$ & $7(7.14 \%)$ & $10(3.37 \%)$ \\
\hline $30-39$ & $6(14.29 \%)$ & $6(10.17 \%)$ & $18(18.37 \%)$ & $77(25.93 \%)$ \\
\hline $40-49$ & $7(16.67 \%)^{* *}$ & $19(32.20 \%)^{*}$ & $18(18.37 \%)^{\text {ss }}$ & $88(29.63 \%)^{\text {s }}$ \\
\hline $50-59$ & $17(40.48 \%)$ & $20(33.90 \%)$ & $36(36.73 \%)$ & $87(29.29 \%)$ \\
\hline$\geq 60$ & $8(19.05 \%)$ & $10(16.95 \%)$ & $19(19.39 \%)$ & $35(11.78 \%)$ \\
\hline
\end{tabular}

${ }^{\star}$ Compared with ${ }^{* *} p=0.07838,{ }^{\$}$ compared with ${ }^{\$ \$} p=0.029125$.

The proportion of normal TG patients was higher in the lean NAFLD group ( $p=0.0008)$, while the proportion of elevated TG patients was higher in the obese NAFLD group ( $p=0.0068)$.

\section{Comparison of serum levels of $\mathrm{CHOL}$ between the lean NAFLD group and the obese NAFLD group}

To avoid the effects of TG, 176 cases of NAFLD patients with normal TG were chosen to analyse the difference of $\mathrm{CHOL}$ level between the lean NAFLD group and the obese NAFLD group. No statistically significant difference in the proportion of elevated $\mathrm{CHOL}$ patients was found between the two groups ( $p=0.8715$ ) (Table IV).

\section{Comparison of serum levels of blood}

glucose between the lean NAFLD group and the obese NAFLD group

The proportion of elevated fasting glucose patients in the obese NAFLD group was higher than that in the lean NAFLD group (18.48\% vs. $8.91 \%$, $p=0.0209$ ) (Table V).

In the lean NAFLD group there was a statistically significant difference in gender distribution among different fasting glucose level groups. The proportion of females was higher in the normal fasting glucose group $(90.48 \%$ vs. $74.58 \%$, $p=0.0209$ )

\section{Discussion}

Nonalcoholic fatty liver disease has become the most common chronic liver disease in the world, which not only damages the liver, but also pro-

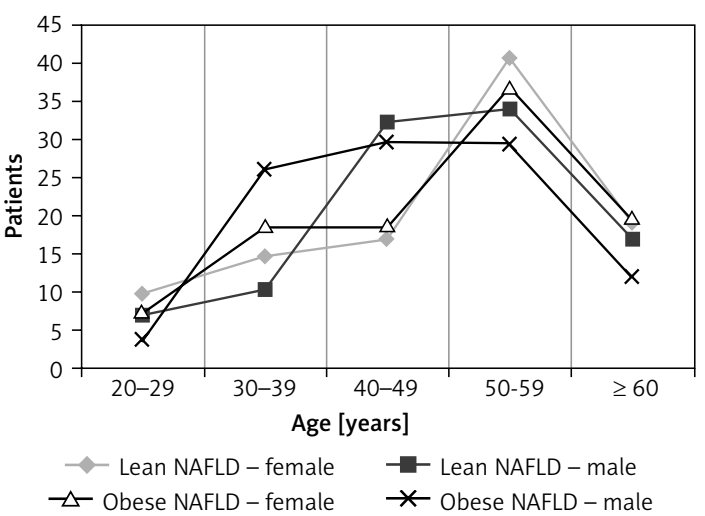

Figure 1. Comparison of age distribution between the lean NAFLD group and the obese NAFLD group

motes the progression of other diseases such as cardiovascular disease and metabolic syndrome. The prevalence of NAFLD is different between lean and obese people, but the current study on NAFLD mainly focuses on the obese population and the lack of lean people. In this paper, the clinical characteristics of lean NAFLD patients were analysed based on indexes such as gender, age, serum levels of TG, CHOL, glucose, and ALT.

It has been reported that NAFLD more often affects men, and premenopausal women are equally protected against developing NAFLD as they are from developing cardiovascular disease [3]. Our results showed that the proportion of males was higher than females in both the lean NAFLD and obese NAFLD groups, but this male-dominated discrepancy of gender distribution was more evident in the obese NAFLD group. 50-59 years was the peak age of both lean and obese NAFLD, and no significant difference in gender distribution

Table III. Comparison of serum levels of TG between the lean NAFLD group and the obese NAFLD group

\begin{tabular}{|c|c|c|}
\hline Parameter & Lean NAFLD $(n=43)$ & Obese NAFLD $(n=259)$ \\
\hline Normal TG $(<1.7 \mathrm{mmol} / \mathrm{l})$ & $29(62.44 \%)^{\star *}$ & $104(40.15 \%)^{*}$ \\
\hline Normal high limit TG (1.7 2.25 mmol/l) & $7(16.28 \%)$ & $58(22.39 \%)$ \\
\hline Elevated TG (> $2.25 \mathrm{mmol} / \mathrm{l})$ & 7 (16.28\%) \#\# & $97(37.45 \%)^{\#}$ \\
\hline
\end{tabular}


Table IV. Comparison of serum levels of $\mathrm{CHOL}$ between the lean NAFLD group and the obese NAFLD group

\begin{tabular}{|lcc|}
\hline CHOL & $\begin{array}{c}\text { Lean NAFLD } \\
(n=46)\end{array}$ & $\begin{array}{c}\text { Obese NAFLD } \\
(n=130)\end{array}$ \\
\hline Normal & $38(82.61 \%)$ & $106(81.54 \%)$ \\
\hline Elevated & $8(17.39 \%)$ & $24(18.46 \%)$ \\
\hline$P=0.8715$. & &
\end{tabular}

was found in this age group. While gender discrepancy was found in the 40-49-year-old age group, the proportion of males was higher than that of females regardless of lean NAFLD or obese NAFLD. Changes in menopausal hormone levels may be responsible for these results. It has been suggested that $17 \beta$-oestradiol (E2) appears to downregulate hepatic lipogenesis in humans, and women often gain weight during menopause because of the dramatic reduction of circulating E2, which may only exaggerate the rise in overweight and obesity, particularly in older adults.

Dyslipidaemia is a common metabolic feature in individuals with NAFLD. The relationship between dyslipidaemia and NAFLD has been a hot topic in recent years, but the characteristics of serum lipid profile in lean NAFLD patients were seldom reported. Dyslipidaemia is characterised by increased triglycerides, decreased HDL cholesterol, and increased LDL cholesterol [4]. To avoid the interaction between different blood lipid components, 302 cases of NAFLD patients with normal CHOL and LDL-C and 176 cases of NAFLD patients with normal TG were chosen individually to analyse the difference of TG and CHOL level between the lean NAFLD group and the obese NAFLD group. We found that elevated TG patients was higher in the obese NAFLD group, while no statistically significant difference in the proportion of elevated CHOL patients was found between the two groups. Hypertriglyceridaemia itself may play a pathogenic role in the development of NAFLD, but there is no highly persuasive evidence. Kim et al. found that hypertriglyceridaemia was an independent predictor for both the development and prognosis of NAFLD and suggested that serum triglyceride level could be an early marker for the progression of NAFLD, especially in lean people [5].

NAFLD is a cause and effect of insulin resistance (IR) [6]. To understand if lean NAFLD also has an effect on blood glucose level, the proportion of patients with different blood glucose levels in the two groups was compared - as expected, we found that the proportion of elevated fasting glucose patients in the obese NAFLD group was higher than that in the lean NAFLD group. More interestingly, in the lean NAFLD group, there was a statistically significant difference in gender distribution among different fasting glucose level groups - the proportion of females was higher in the normal fasting glucose group, which may be related to sexual dimorphism in abnormal metabolism in NAFLD. When we compare the abnormal rate of ALT between lean and obese NAFLD groups, we found that elevated ALT was more common in the obese NAFLD group, which suggests a higher risk of development of NASH and maybe worse outcome in overweight/obese NAFLD patients.

In conclusion, sexual dimorphism exists in lean NAFLD patients, but this trend was most pronounced during the stage of 40-49-year-old age group and disappeared after entering the 50-59-year-old stage. In lean NAFLD patients, normal levels of TG and blood glucose were more common, and the occurrence of NASH was less common than in obese NAFLD patients. Although the data covered in this study are limited, these results may provide some information that is useful in expanding our understanding of the pathogenesis and providing hints for prevention and treatment of NAFLD.

\section{Conflict of interest}

The authors declare no conflict of interest.

Table V. Comparison of serum levels of blood glucose between the lean NAFLD group and the obese NAFLD group

\begin{tabular}{|lcccccc|}
\hline Parameter & \multicolumn{3}{c}{ Lean NAFLD } & \multicolumn{3}{c|}{ Obese NAFLD } \\
\cline { 2 - 7 } & $\begin{array}{c}\text { Total } \\
(n=101)\end{array}$ & $\begin{array}{c}\text { Female } \\
(n=42)\end{array}$ & $\begin{array}{c}\text { Male } \\
(n=59)\end{array}$ & $\begin{array}{c}\text { Total } \\
(n=395)\end{array}$ & $\begin{array}{c}\text { Female } \\
(n=98)\end{array}$ & $\begin{array}{c}\text { Male } \\
(n=297)\end{array}$ \\
\hline $\begin{array}{l}\text { Elevated fasting } \\
\text { glucose } \\
(\geq 7.0 \mathrm{mmol} / \mathrm{l})\end{array}$ & $9^{\# \#}(8.91 \%)$ & $2(4.76 \%)$ & $7(11.86 \%)$ & $73^{\#}(18.48 \%)$ & $19(19.39 \%)$ & $54(18.18 \%)$ \\
\hline $\begin{array}{l}\text { Impaired } \\
\text { fasting glucose } \\
(6.1 \sim 6.9 \mathrm{mmol} / \mathrm{l})\end{array}$ & $10(9.90 \%)$ & $2(4.76 \%)$ & $8(13.56 \%)$ & $49(12.41 \%)$ & $14(14.29 \%)$ & $35(11.78 \%)$ \\
\hline $\begin{array}{l}\text { Normal } \\
\text { fasting glucose } \\
(<6.1 \mathrm{mmol} / \mathrm{l})\end{array}$ & $82(81.19 \%)$ & $38(90.48 \%)^{* *}$ & $44(74.58 \%)^{*}$ & $273(69.11 \%)$ & $65(66.33 \%)$ & $208(70.03 \%)$ \\
\hline${ }^{\# \text { Compared with }{ }^{\# \#} p=0.0209,{ }^{*} \text { compared with }{ }^{* *} p=0.0439 .}$ & & & & \\
\hline
\end{tabular}




\section{References}

1. Schiffer L, Kempegowda P, Arlt W, O'Reilly MW. Mechanisms in endocrinology: the sexually dimorphic role of androgens in human metabolic disease. Eur J Endocrinol 2017; 177: R125-43.

2. Jian-gao F. Guidelines for management of nonalcoholic fatty liver disease: an updated and revised edition. Zhonghua Gan Zang Bing Za Zhi 2010; 18: 163-6.

3. Nishioji K, Sumida Y, Kamaguchi M, et al. Prevalence of and risk factors for non-alcoholic fatty liver disease in a non-obese Japanese population, 2011-2012. J Gastroenterol 2015; 50: 95: 108.

4. Targher G, Day CP, Bonora E. Risk of cardiovascular disease in patients with nonalcoholic fatty liver disease. N Engl J Med 2010; 363: 1341-50.

5. Kim NH, Kim JH, Kim YJ, et al. Clinical and metabolic factors associated with development and regression of nonalcoholic fatty liver disease in nonobese subjects. Liver Int 2014; 34: 604-11.

6. Asrih M, Jornayvaz FR. Metabolic syndrome and nonalcoholic fatty liver disease: Is insulin resistance the link? Mol Cell Endocrinol 2015; 418: 55-65. 